Pacific Journal of Mathematics

ON THE CARDINALITY RELATIONSHIPS BETWEEN 


\title{
ON THE CARDINALITY RELATIONSHIPS BETWEEN DISCRETE COLLECTIONS AND OPEN COVERS
}

\author{
JAMES R. BOONE
}

\begin{abstract}
This paper unifies and extends various theorems which deal with the relationship between the cardinality of discrete collections and the cardinality of open coverings. For this purpose, the class of spaces which are irreducible of order $\alpha$ is defined. This class includes the $\delta \theta$-refinable and the $[\alpha, \infty)$-refinable spaces. Some examples of applications of this class are: a space is $[\alpha, x)$-compact if and only if it is irreducible of order $\alpha$ and has the $\alpha-B W$ property and if $X$ is irreducible of order $\Delta(X)$, then $\Delta(X) \leqq L(X) \leqq \Delta(X)^{+}$. The open ordinal space $[0, \Omega)$ serves as a model to generate examples of spaces which are irreducible of order $\alpha$ but not irreducible of order $\beta$, if $\beta>\alpha$.
\end{abstract}

1. Preliminaries. Various theorems have been presented which relate the cardinality of open covers to the cardinality of discrete collections of closed sets. In the theorem of Alexandroff and Urysohn [1] which states that a space $X$ is $[\alpha, \beta]$-compact ${ }^{r}$ if and only if every open covering of $X$ whose cardinality is a regular cardinal in $[\alpha, \beta]$ has a subcover of smaller cardinality, the origin is indicted for the notion of a space in which every open cover has a refinement which covers the space minimally. Such spaces have been called irreducible [5] and [6] and minimal cover refinable [7]. Arens and Dugundji [3] used the irreducibility of metacompact spaces to prove that a space is compact if and only if it is countably compact and metacompact. More recently, Aquaro [2] proved that every point-countable open cover of a space, in which every discrete collection of closed sets is countable, has a countable subcover. Aull [4] has generalized Aquaro's "meta-Lindelöf" condition with the concept of a $\delta \theta$-refinable space and shown that $\boldsymbol{N}_{1}$-compact, $\delta \theta$-refinable spaces are Lindelöf. Hodel and Vaughan [9] have extended these results by the use of more general spaces, which they call $[\alpha, \beta]$-refinable.

2. Introducion. It is the purpose of this paper to introduce a more general version of irreducibility which will unify most of the results which relate the cardinality of open covers and the cardinality of discrete collections of closed sets. Although irreducibility plays a major role in unifying a class of theorems dealing with these cardinality relationships, a 
more general version appears to be natural in dealing with other theorems. In particular, it has been shown that $\theta$-refinable [5] and weak $\bar{\theta}$-refinable [6] spaces are irreducible. In these spaces we are dealing with conditions which are essentially "point-finite". However, as Aull [4] has shown, important cardinality theorems are also true for the large class of $\delta \theta$-refinable spaces and Hodel and Vaughan [9] have generalized these to $[\alpha, \beta]$-refinable spaces. In [5] irreducibility was characterized as follows:

THEOREM 2.1. [5] A space $X$ is irreducible if and only if for every open cover $U=\left\{U_{a}: a \in A\right\}$ there exists a discrete collection of closed sets $\mathscr{T}=\left\{T_{b}: b \in B\right\}$ such that $B \subset A, T_{b} \subset U_{b}$ for each $b \in B$ and $\left\{U_{b}: b \in\right.$ $B\}$ covers $X$.

The requirement that each $T_{b}$ be in one of the sets from the original open cover and then that $\left\{U_{b}: b \in B\right\}$ cover the space is too restrictive in considering spaces which allow the order, of the open refinement at points, to have infinite cardinality. To include this class of spaces, in which "point-finiteness" is not guaranteed, for every infinite cardinal $\alpha$, we make the following definition; which is motivated by Theorem 2.1, $\delta \theta$-refinable and $[\alpha, \infty)$-refinable spaces.

Definition. A topological space $X$ is irreducible of order $\alpha$ provided: for every open covering $\mathcal{U}$ of $X$ there exists an open refinement $\mathscr{V}=\cup\left\{\mathscr{V}_{a}: a \in A\right\}$ of $\mathcal{U}$ and a family of discrete closed collections $\left\{\mathscr{T}_{a}: a \in A\right\}$ where card $(A)<\alpha$ such that:

(1) for each $T \in \mathscr{T}_{a}, \mathscr{V}_{T}=\left\{V \in \mathscr{V}_{a}: T \subset V\right\}$ is nonempty and card $\left(\mathscr{V}_{T}\right)<\alpha$,

(2) $\left\{V: V \in \mathscr{V}_{T}, T \in \mathscr{T}_{a}, a \in A\right\}$ covers $X$.

From the definition, if $\alpha$ and $\beta$ are infinite cardinals, irreducible implies irreducible of order $\alpha$ and if $\alpha \leqq \beta$ then irreducible of order $\alpha$ implies irreducible of order $\beta$.

In $\S 3$, the relationship between $[\alpha, \infty)$-refinable spaces and spaces which are irreducible of order $\alpha$ is presented. Applications of irreducible of order $\alpha$, to the cardinality relationships between discrete collections and open coverings, are given in $\S 4$. Section 5 contains an example which shows that if $\beta>\alpha$, then irreducible of order $\beta$ does not imply irreducible of order $\alpha$.

All spaces will be assumed to be $T_{1}$-spaces. Infinite cardinals will be denoted by lower case Greek letters or alephs. If $\mathcal{U}$ is a collection of subsets of a space $X$ and $p \in X$, ord $(p, \mathcal{U})$ will denote the cardinality of the subcollection of $\mathcal{U}$ consisting of those sets which contain $p$. 
3. On $[\alpha, \infty)$-refinable spaces. Some of the results of Aull [4] play an essential role in this study and they are restated here for completeness. A subset $M$ of a space $X$ is called distinguished with respect to an open cover $\mathcal{U}$ if for each pair of distinct points $x, y \in M$, if $x \in U \in \mathcal{U}$ then $y \notin U$. Also, $M$ is maximally distinguished with respect to $U$ on a set $H$ if $M \subset H, M$ is distinguished and if $P$ is distinguished and $M \subset P \subset H$, then $P=M$. Sets of this type have also been used extensively by Hodel [8] and Hodel and Vaughan [9].

LEMMA 3.1. [Aull]

(1) A distinguished set is a discrete collection of singletons.

(2) If a distinguished set $M$ with respect to an open cover $U$ is contained in a set $H$, then it is contained in a maximally distinguished set on $H$ with respect to $U$.

(3) If $\mathscr{V}$ is the subcollection of an open cover $U$ that intersects a maximally distinguished set $M$ with respect to $\mathcal{U}$ on a set $H$, then $\mathscr{V}$ covers $H$.

Hodel and Vaughan [9] have unified many diverse theorems involving cardinality conditions for open coverings in their paper on $[\alpha, \beta]$-compactness. Four types of refinements were introduced in [9]. We will consider the $[\alpha, \infty)$-refinable space, because the necessary variations of irreducible of order $\alpha$ which would lend themselves to other applications in [9] will be indicated by the following results. A space $X$ is $[\alpha, \infty)$-refinable [9], provided: If $\mathcal{U}$ is any open covering of $X$ there exists a family $\left\{\mathscr{V}_{a}: a \in A\right\}$ of open refinements of $\mathcal{U}$, each of which cover $X$, such that $\operatorname{card}(A)<\alpha$ and for each $p \in X$, there exists an $a \in A$ such that $\operatorname{ord}\left(p, \mathscr{V}_{a}\right)<\alpha$.

THEOREM 3.2. Every $[\alpha, \infty)$-refinable space is irreducible of order $\alpha$.

Proof. Let $U$ be an open covering of an $[\alpha, \infty)$-refinable space $X$. Let $\mathscr{V}=\cup\left\{\mathscr{V}_{a}: a \in A\right\}$ be an open refinement such that $\mathscr{V}_{a}$ covers $X$ for each $a \in A, \operatorname{card}(A)<\alpha$ and for each $p \in X$ there is an $a \in A$ such that $\operatorname{ord}\left(p, \mathscr{V}_{a}\right)<\alpha$. For each $a \in A$, let $H_{a}=\left\{p \in X: \operatorname{ord}\left(p, \mathscr{V}_{a}\right)<\right.$ $\alpha$. Let $M_{a}$ be a maximally distinguished set in $H_{a}$ relative to the open covering $\mathscr{V}_{a}$. Then, by Lemma $3.1, M_{a}$ is a discrete collection of singletons and $\left\{V \in \mathscr{V}_{a}: V \cap M_{a} \neq \varnothing\right\}$ covers $H_{a}$. Let $\mathscr{T}_{a}=$ $\left\{\{p\}: p \in M_{a}\right\}$. Since ord $\left(p, \mathscr{V}_{a}\right)<\alpha$ for each $p \in M_{a}, \mathscr{V}_{p}=$ $\left\{V \in \mathscr{V}_{a}: p \in V\right\}$ is nonempty and card $\left(\mathscr{V}_{p}\right)<\alpha$. Since $X=$ $\cup\left\{H_{a}: a \in A\right\},\left\{V: V \in \mathscr{V}_{p},\{p\} \in \mathscr{T}_{a}, a \in A\right\}$ covers $X$. This completes the proof.

Since the $\delta \theta$-refinable spaces of Aull [4] are precisely the $\left[\boldsymbol{N}_{1}, \infty\right)$ refinable spaces, the following corollary is true. 
COROllary 3.3. Every $\delta \theta$-refinable space is irreducible of order $\boldsymbol{\aleph}_{1}$.

The variations of "irreducible of order $\alpha$ ", such as irreducible of order $[\alpha, \beta]$ and irreducible of order $[\alpha, \beta]^{r}$ which would yield modifications of theorems in [9] involving $[\alpha, \beta]$-compact, $[\alpha, \beta]$-compact ${ }^{r},[\alpha, \beta]$ refinable, $[\alpha, \beta]$-refinable ${ }^{r}$ and $[\alpha, \infty]$-refinable' could be defined in the obvious manner.

4. Applications of irreducibility of order $\alpha$. A space $X$ has the $\alpha-B W$ property [9] if every subset $M$ of $X$ with card $(M) \geqq \alpha$ has an $\omega$-accumulation point. In $T_{1}$-spaces it suffices for such sets to have a cluster point. Singular cardinals are characterized by Jech [10, Lemma 11] in the following manner: a cardinal $\kappa$ is singular if and only if there exists a cardinal $\lambda<\kappa$ and a family $\left\{S_{\xi}: \xi<\lambda\right\}$ of subsets of $\kappa$ such that $\kappa=\cup\left\{S_{\xi}: \xi<\kappa\right\}$ and card $\left(S_{\xi}\right)<\kappa$, for each $\xi<\lambda$. This characterization is stated here to clarify the need, in the following theorems, to restrict our attention to cardinals which are not singular; that is, regular cardinals.

THEOREM 4.1. Let $\alpha$ be a regular cardinal. A space $X$ is $[\alpha, \infty)$ compact if and only if $X$ is irreducible of order $\alpha$ and has the $\alpha-B W$ property.

Proof. Since the necessity is clearly valid, we will prove only the sufficiency. Let $\mathcal{u}$ be an open covering of a space which is irreducible of order $\alpha$ and has the $\alpha-B W$ property. Let $\mathscr{V}=\cup\left\{\mathscr{V}_{a}: a \in A\right\}$ be an open refinement of $\mathcal{U}$, and let $\left\{\mathscr{T}_{a}: a \in A\right\}$ be a family of discrete closed collections where $\operatorname{card}(A)<\alpha$ such that for each $T \in \mathscr{T}_{a}, \mathscr{V}_{T}=$ $\left\{V \in \mathscr{V}_{a}: T \in V\right\}$ is nonempty and $\operatorname{card}\left(\mathscr{V}_{T}\right)<\alpha$ and $\left\{V: V \in \mathscr{V}_{T}\right.$, $\left.T \in \mathscr{T}_{a}, a \in A\right\}$ covers the space. Since the space has the $\alpha-B W$ property, for each $a \in A$ card $\left(\mathscr{T}_{a}\right)<\alpha$. Since $\alpha$ is a regular cardinal, $\left\{V: V \in \mathscr{V}_{T}, T \in \mathscr{T}_{a}, a \in A\right\}$ has cardinality less than $\alpha$. Accordingly, the space is $[\alpha, \infty)$-compact.

COROllary 4.2. A space is $\left[\boldsymbol{N}_{1}, \infty\right)$-compact ( $\equiv$ Lindelöf) if and only if it is irreducible of order $\boldsymbol{N}_{1}$ and has the $\boldsymbol{N}_{1}-B W$ property $\left(\equiv \boldsymbol{N}_{1}\right.$ compact).

Since $\boldsymbol{\aleph}_{1}-B W$ implies $\boldsymbol{\aleph}_{1}-B W$, Corollary 4.2 provides the intermediate step for the next corollary to Theorem 4.1. This corollary indicates the sensitivity of a strengthening of the $B W$ property, while the weaker form of irreducibility is preserved. In particular, a countably compact space which is irreducible or order $\boldsymbol{N}_{1}$ is compact. (That is, irreducible of order $\boldsymbol{N}_{0}$ is not required.) 
Corollary 4.3. A space is $\left[\mathbf{N}_{0}, \infty\right)$-compact (三compact) if and only if it is irreducible of order $\boldsymbol{N}_{1}$ and has the $\boldsymbol{N}_{0}-B W$ property ( $\equiv$ countably compact).

Corollary 4.4. Let $\alpha$ be a regular cardinal. A space $X$ is $[\alpha, \beta]$ compact if and only if $X$ is irreducible of order $[\alpha, \beta]$ and has the $\alpha-B W$ property.

The Lindelöf degree, introduced by Juhasz [11], of a space $X$ is denoted by $L(X)$ and defined to be $\boldsymbol{N}_{0} \cdot \alpha$ where $\alpha$ is the least cardinal such that every open cover of $X$ has a subcover of cardinality $\leqq \alpha$. The proof of the following theorem is contained in the proof of Theorem 4.1, since $[\alpha, \infty)$-compact implies $L(X) \leqq \alpha$.

THEOREM 4.5. Let $\alpha$ be a regular cardinal. If $X$ is irreducible of order $\alpha$ and has the $\alpha-B W$ property, then $L(X) \leqq \alpha$.

Hodel defines the discreteness character [8] of a space $X$ to be $\mathbf{N}_{0} \cdot \alpha$, where $\alpha=\sup \{\operatorname{card}(\mathscr{F}): \mathscr{F}$ is a discrete collection of nonempty closed sets in $X\}$. The discreteness character of $X$ is denoted by $\Delta(X)$. Clearly, $\Delta(X) \leqq L(X)$ for all spaces $X$. Also, if $D$ is any distinguished set $\operatorname{card}(D) \leqq \Delta(X) \leqq L(X)$.

Lemma 4.6. If $\Delta(X)=\alpha$, then $X$ has the $\alpha^{+}-B W$ property.

Proof. Suppose $X$ does not have the $\alpha^{+}-B W$ property. Let $H$ be any set such that card $(H) \geqq \alpha^{+}$and $H$ has no cluster points. Then $\{\{p\}: p \in H\}$ is a discrete collection of closed sets with cardinality $\geqq \alpha^{+}$. Then $\Delta(X)>\alpha$.

THEOREM 4.7. Let $\alpha$ be a regular cardinal. If $X$ is irreducible of order $\alpha$ and $\Delta(X)=\alpha$, then $\alpha=\Delta(X) \leqq L(X) \leqq \alpha^{+}$.

Proof. Since $X$ is irreducible of order $\alpha$, then $X$ is irreducible of order $\alpha^{+}$and from the preceding lemma $\Delta(X)=\alpha$ implies $X$ has the $\alpha^{+}-B W$ property. By Theorem $4.5, L(X) \leqq \alpha^{+}$. Thus $\alpha=\Delta(X) \leqq$ $L(X) \leqq \alpha^{+}$.

Since irreducibility of order $\boldsymbol{N}_{0}$ implies irreducibility of $\operatorname{order} \boldsymbol{N}_{1}$ and $\Delta(X)=\boldsymbol{N}_{0}$ implies $\boldsymbol{N}_{1}$-compact, Corollary 4.2 yields the following variation of Theorem 4.7 .

Corollary 4.8. $X$ is irreducible of order $\boldsymbol{N}_{1}$ and $\Delta(X)=\boldsymbol{N}_{0}$ if and only if $L(X)=\mathbf{N}_{0}$. 


\section{Example.}

EXAMPLE 5.1. If $\beta>\alpha$, then there exists a space which is irreducible of order $\beta$ which is not irreducible of order $\alpha$.

Since an irreducible of order $\boldsymbol{N}_{1}$, countably compact space is compact, $[0, \Omega)$ is not irreducible of order $\boldsymbol{N}_{1} .([0, \Omega)$ is the space of countable ordinals with the interval topology.) Let $\mathcal{U}$ be any open covering of $[0, \Omega)$ by basic open sets. Let $M$ be a maximally distinguished set relative to $\mathcal{U}$. Then $M$ is finite. Suppose ord $(p, \mathcal{U}) \leqq \boldsymbol{N}_{0}$, for each $p \in M$. Since each basic open set is countable, $U\{U: U \cap M \neq \varnothing\}$ is countable and thus does not cover $[0, \Omega)$. Thus for some $p_{0} \in M$, $\operatorname{ord}\left(p_{0}, \mathscr{U}\right)>\boldsymbol{N}_{0}$. Since every point has order $\leqq \boldsymbol{N}_{1}$, ord $\left(p_{0}, \mathscr{U}\right)=\boldsymbol{N}_{1}$. Hence $\left\{\beta: p_{0} \in(\alpha, \beta] \in \mathcal{U}\right\}$ is cofinal in $[0, \Omega)$, and $[0, \Omega)-\operatorname{st}\left(p_{0}, \mathcal{U}\right)$ is compact, and contains $M-\left\{p_{0}\right\}$. Note that $p_{0}$ is the only point in $M$ which has uncountable order relative to $U$. That is, for each $p \in M-$ $\left\{p_{0}\right\}$, ord $(p, \mathcal{U}) \leqq \boldsymbol{N}_{0}$. Thus $[0, \Omega)$ is irreducible of order $\boldsymbol{N}_{2}$, but not irreducible of order $\boldsymbol{\aleph}_{1}$. Accordingly, $[0, \Omega)$ is an example of an $\boldsymbol{\aleph}_{1}$ compact, irreducible of order $\boldsymbol{N}_{2}$ space which is not Lindelöf, and a countably compact, irreducible or order $\boldsymbol{\aleph}_{2}$ space which is not compact. Hence the irreducibility in Corollaries 4.2 and 4.3 can not be relaxed to $\boldsymbol{N}_{1}^{+}=\boldsymbol{N}_{2}$. Examples can be constructed to show: if $\beta>\alpha$ then irreducible of order $\beta$ does not imply irreducible or order $\alpha$ by considering open ordinal spaces of appropriately large cardinality.

\section{REFERENCES}

1. P. S. Alexandroff and P. Urysohn, Memoire sur les espaces topologiques compacts, Verh. Kon. Akad. Van Weten. Te Amsterdam, XIV (1929), 1-96.

2. G. Aquaro, Point countable open coverings in countably compact spaces, General Topology and Its Relations to Modern Analysis and Algebra II, Academia, Prague (1966), 39-41.

3. R. Arens and J. Dugundji, Remark on the concept of compactness, Portugaliae Math., 9 (1950), 141-143.

4. C. E. Aull, A generalization of a theorem of Aquaro, Bull. Austral. Math. Soc., 9 (1973), 105-108.

5. J. R. Boone, On irreducible spaces, Bull. Austral. Math. Soc., 12 (1975), 143-148.

6. - On irreducible spaces II, Pacific J. Math.

7. U. J. Christian, A note on the relationship between Lindelöf and $\boldsymbol{\aleph}_{1}$-compact spaces, Comment. Math. Prace Mat., 16 (1972), 215-217.

8. R. E. Hodel, On a theorem of Arhangelskii concerning Lindelöf p-spaces, Canad. J. Math., 27 (1975), 459-468.

9. R. E. Hodel and J. E. Vaughan, A note on $[a, b]$-compactness, Gen. Topology Appl., 4 (1974), 179-189.

10. T. J. Jech, Lectures in set theory with particular emphasis on the method of forcing, Lecture Notes in Mathematics, No. 217, Springer-Verlag (1971).

11. I. Juhasz, Cardinal functions in topology, Math. Centre Tracts, No. 34, Amsterdam (1971).

Received May 8, 1975 and in revised form November 12, 1975.

TEXAS A \& M UNIVERSITY 


\section{PACIFIC JOURNAL OF MATHEMATICS}

\section{EDITORS}

RICHARD ARENS (Managing Editor)

University of California

Los Angeles, California 90024

\section{J. Dugundu}

Department of Mathematics University of Southern California Los Angeles, California 90007

D. Gilbarg and J. Milgram Stanford University Stanford, California 94305

\section{ASSOCIATE EDITORS}
E. F. BECKENBACH
B. H. NeumanN
F. WOLF
K. YoSHIDA

\section{SUPPORTING INSTITUTIONS}

UNIVERSITY OF BRITISH COLUMBIA CALIFORNIA INSTITUTE OF TECHNOLOGY

UNIVERSITY OF CALIFORNIA

MONTANA STATE UNIVERSITY

UNIVERSITY OF NEVADA

NEW MEXICO STATE UNIVERSITY

OREGON STATE UNIVERSITY

UNIVERSITY OF OREGON

OSAKA UNIVERSITY

\author{
UNIVERSITY OF SOUTHERN CALIFORNIA \\ STANFORD UNIVERSITY \\ UNIVERSITY OF HAWAII \\ UNIVERSITY OF TOKYO \\ UNIVERSITY OF UTAH \\ WASHINGTON STATE UNIVERSITY \\ UNIVERSITY OF WASHINGTON \\ AMERICAN MATHEMATICAL SOCIETY
}

The Supporting Institutions listed above contribute to the cost of publication of this Journal, but they are not owners or publishers and have no responsibility for its contents or policies.

Mathematical papers intended for publication in the Pacific Journal of Mathematics should be in typed form or offset-reproduced (not dittoed), double spaced with large margins. Underline Greek letters in red, German in green, and script in blue. The first paragraph or two must be capable of being used separately as a synopsis of the entire paper. Items of the bibliography should not be cited there unless absolutely necessary, in which case they must be identified by author and Journal, rather than by item number. Manuscripts, in duplicate, may be sent to any one of the four editors. Please classify according to the scheme of Math. Reviews, Index to Vol. 39. All other communications should be addressed to the managing editor, or Elaine Barth, University of California, Los Angeles, California, 90024.

100 reprints are provided free for each article, only if page charges have been substantially paid. Additional copies may be obtained at cost in multiples of 50 .

The Pacific Journal of Mathematics is issued monthly as of January 1966. Regular subscription rate: $\$ 72.00$ a year (6 Vols., 12 issues). Special rate: $\$ 36.00$ a year to individual members of supporting institutions.

Subscriptions, orders for back numbers, and changes of address should be sent to Pacific Journal of Mathematics, 103 Highland Boulevard, Berkeley, California, 94708.

PUBLISHED BY PACIFIC JOURNAL OF MATHEMATICS, A NON-PROFIT CORPORATION

Printed at Jerusalem Academic Press, POB 2390, Jerusalem, Israel.

$$
\begin{gathered}
\text { Copyright } 1976 \text { Pacific Journal of Mathematics } \\
\text { All Rights Reserved }
\end{gathered}
$$




\section{Pacific Journal of Mathematics}

Vol. 62, No. 2

February, 1976

Allan Russell Adler and Catarina Isabel Kiefe, Pseudofinite fields, procyclic

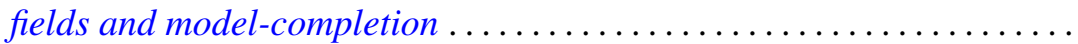

Christopher Allday, The stratification of compact connected Lie group

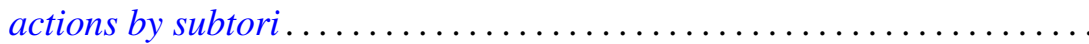

Martin Bartelt, Commutants of multipliers and translation operators .......

Herbert Stanley Bear, Jr., Ordered Gleason parts ..................

James Robert Boone, On irreducible spaces. II .....................

James Robert Boone, On the cardinality relationships between discrete

collections and open covers ............................

L. S. Dube, On finite Hankel transformation of generalized functions .......

Michael Freedman, Uniqueness theorems for taut submanifolds . . . . . . . . .

Shmuel Friedland and Raphael Loewy, Subspaces of symmetric matrices

containing matrices with a multiple first eigenvalue .............

Theodore William Gamelin, Uniform algebras spanned by Hartogs

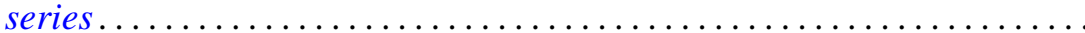

James Guyker, On partial isometries with no isometric part ............

Shigeru Hasegawa and Ryōtarō Satō, A general ratio ergodic theorem for



Nigel Kalton and G. V. Wood, Homomorphisms of group algebras with norm less than $\sqrt{2}$.

Thomas Laffey, On the structure of algebraic algebras...

Will Y. K. Lee, On a correctness class of the Bessel type differential operator $S_{\mu}$

Robert D. Little, Complex vector fields and divisible Chern classes ....

Kenneth Louden, Maximal quotient rings of ring extensions . .

Dieter Lutz, Scalar spectral operators, ordered $l^{\rho}$-direct sums, and the

counterexample of Kakutani-McCarthy . .

Ralph Tyrrell Rockafellar and Roger Jean-Baptiste Robert Wets, Stochastic

convex programming: singular multipliers and extended duality

singular multipliers and duality.

Edward Barry Saff and Richard Steven Varga, Geometric overconvergence of rational functions in unbounded domains ..........

Joel Linn Schiff, Isomorphisms between harmonic and P-harmonic Hardy

spaces on Riemann surfaces.

Virinda Mohan Sehgal and S. P. Singh, On a fixed point theorem of

Krasnoselskii for locally convex spaces.

Lewis Shilane, Filtered spaces admitting spectral sequence operations

Michel Smith, Generating large indecomposable continua . 